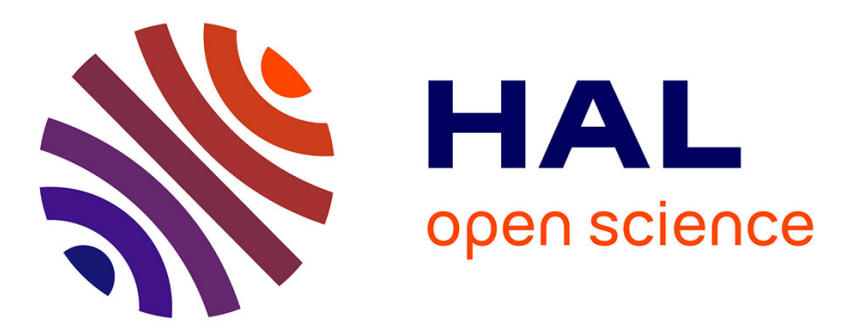

\title{
Contribution à l'étude du champ magnétique et des courants induits dans une bande conductrice mince de dimensions finies
}

\author{
A. Gagliano, M. Kant, A. Mouillet
}

\section{- To cite this version:}

A. Gagliano, M. Kant, A. Mouillet. Contribution à l'étude du champ magnétique et des courants induits dans une bande conductrice mince de dimensions finies. Revue de Physique Appliquée, 1972, 7 (4), pp.253-260. 10.1051/rphysap:0197200704025300 . jpa-00243626

HAL Id: jpa-00243626

https://hal.science/jpa-00243626

Submitted on 1 Jan 1972

HAL is a multi-disciplinary open access archive for the deposit and dissemination of scientific research documents, whether they are published or not. The documents may come from teaching and research institutions in France or abroad, or from public or private research centers.
L'archive ouverte pluridisciplinaire HAL, est destinée au dépôt et à la diffusion de documents scientifiques de niveau recherche, publiés ou non, émanant des établissements d'enseignement et de recherche français ou étrangers, des laboratoires publics ou privés. 
05.00,

\title{
CONTRIBUTION A L'ÉTUDE DU CHAMP MAGNÉTIQUE ET DES COURANTS INDUITS DANS UNE BANDE CONDUCTRICE MINCE DE DIMENSIONS FINIES
}

\author{
A. GAGLIANO, M. KANT et A. MOUILlet (*) \\ (Reçu le 16 mars 1972, révisé le 23 juin 1972)
}

\begin{abstract}
Résumé. - On établit analytiquement la distribution des courants induits au sein d'une bande conductrice mince, en mouvement de translation uniforme dans un champ magnétique extérieur d'extension finie purement glissant. On montre que les grandeurs électromagnétiques ne s'expriment pas par des séries, mais par des combinaisons polynomiales exponentielles, à cause des dimensions finies du système. On indique la forme du champ magnétique extérieur permettant d'obtenir un champ d'induction résultant purement glissant à l'intérieur de la bande.

Bien qu'à l'origine de la recherche se trouve l'étude des machines linéaires, le problème est traité d'une façon générale et les résultats obtenus peuvent s'appliquer au calcul de divers dispositifs, comme machines en arc, écrans magnétiques, relais de translation, etc...
\end{abstract}

\begin{abstract}
The distribution of the induced currents in a thin conducting plate moving at constant velocity through a travelling magnetic field of finite extension, is investigated from an analytical point of view. The authors show that the electromagnetic quantities are not expressed by mathematical series, but by exponential polynomial combinations, because of finite dimensions of the system. They give the wave form of the external magnetic field so as to obtain a resulting travelling magnetic field inside the plate.

Even though the purpose of the research is the study of linear machines, the problem has been treated from a theoretical point of view, and the results may be practically applied to the theoretical design of many devices, such as « arch » motors, magnetic shields, etc.
\end{abstract}

I. Introduction. - L'avènement des convertisseurs électromécaniques nouveaux, et en particulier à circuit magnétique ouvert, ou/et à induit constitué par un conducteur massif, est à la base de plusieurs recherches fondamentales, car leur analyse nécessite des méthodes mathématiques plus puissantes que celles utilisées pour les dispositifs classiques régis par des principes physiques similaires. Ainsi l'origine du présent mémoire réside dans l'étude de la machine linéaire à induction qui comprend, schématiquement, un ou deux inducteurs magnétiques plans, portant des enroulements d'excitation et un induit constitué par une bande conductrice (Fig. 1). Par rapport aux machines à

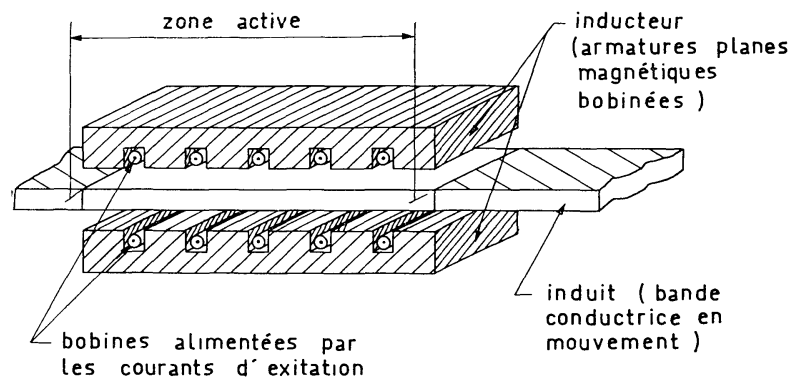

FIG. 1. - Modèle de principe d'un moteur linéaire à induit mobile.

(*) Laboratoire de Génie Electrique des Universités de Paris VI et Paris XI (associé au CNRS) LCIE 33, avenue du Général-Leclerc, 92, Fontenay-aux-Roses. géométrie circulaire, le dispositif étudié se caractérise par une distribution bidimensionnelle des courants induits, et par la présence de champs et de courants de fuites, qui se développent en dehors de la «zone active » où s'effectue la conversion d'énergie. Cette zone correspond pratiquement à la partie de l'induit localisée entre les deux inducteurs.

Afin de pouvoir calculer les caractéristiques d'une telle machine, il convient de déterminer deux paramètres essentiels :

- la forme du champ magnétique résultant,

- la distribution des courants induits.

$\mathrm{Au}$ point de vue théorique, le problème peùt se ramener à l'étude de l'interaction d'un champ magnétique glissant $B_{\mathrm{c}}$ d'extension finie et d'une bande conductrice mince de dimensions finies (Fig. 2).

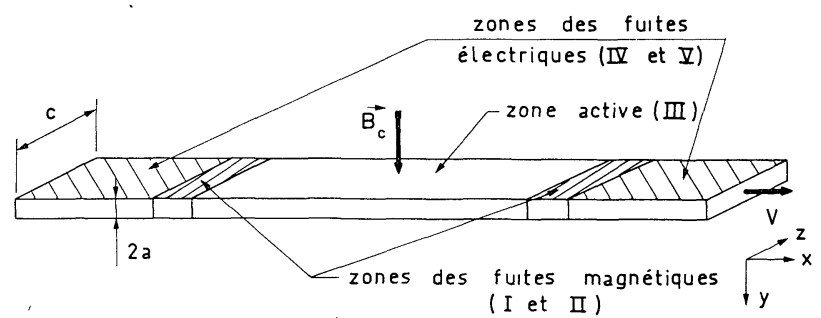

FIg. 2. - Induit du moteur linéaire de la figure 1 assimilé à une bande de dimensions finies divisée en cinq zones longitudinales. 
Un tel problème est généralement traité, dans la littérature, à l'aide d'intégrales de Fourier [1]. Ce procédé n'est toutefois applicable qu'à quelques configurations, caractérisées par des conditions aux limites très simplifiées. Dans notre étude, nous avons choisi une autre méthode d'approche, basée sur le modèle dit «à cinq zones», proposé initialement dans [2], où il a été prouvé que l'étude des champs et des courants peut être effectuée plus facilement, dans une configuration semblable à celle de la figure 2 , lorsqu'on divise longitudinalement (selon $O x$ ) la bande conductrice en cinq parties de caractéristiques différentes :

- une "zone active » III, qui correspond à l'étendue du champ inducteur (et partant, à une certaine densité de courant extérieur à la bande),

- deux zones I et II, situées de part et d'autre de la zone III, dans lesquelles peuvent se développer des phénomènes d'induction provenant des champs de fuites,

- deux zones IV et $\mathrm{V}$, où le champ de fuites est suffisamment amorti pour qu'on puisse le négliger. Dans ces zones, prolongeant la bande conductrice, peuvent circuler des courants de fuites provenant des zones adjacentes.

Les buts de cette « décomposition » sont d'une part, d'assurer un certain nombre de conditions aux limites intermédiaires aux équations du champ électromagnétique, et d'autre part de matérialiser géométriquement, par la longueur des zones, les grandeurs physiques (par exemple, les courants et le champ magnétique de fuites). On peut également remarquer que la distribution des courants dans les zones IV et V dérive uniquement d'un potentiel magnétique scalaire [3].

Dans le cas général, nous ajoutons à toutes les zones, deux bandes latérales immobiles (selon $O z$ ), dites «barres de fermeture », dont la résistivité peut être différente de celle de la bande conductrice, qui facilitent la fermeture des courants induits. Par ailleurs, leur existence permet, en faisant varier leur résistivité, d'englober tous les cas possibles, depuis la bande de largeur infinie selon $O z$ (résistance nulle) jusqu'à la bande où tous les courants se ferment dans la partie médiane (résistance infinie). Le modèle à cinq zones ainsi modifié est schématisé sur la figure 3 . Les diffé-

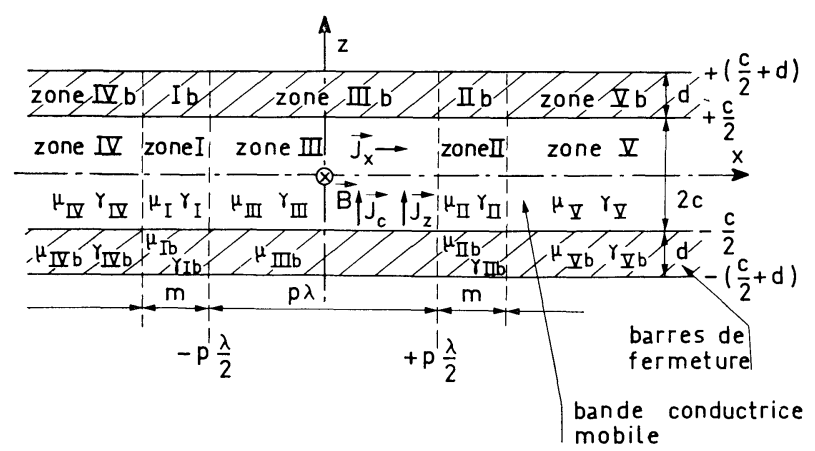

FIG. 3. - Modèle mathématique de la bande de dimensions finies avec les barres de fermeture. rentes zones de la bande sont déterminées par leurs dimensions géométriques et les caractéristiques du milieu (perméabilité magnétique $\mu$ et conductivité $\gamma$, affectées du chiffre romain correspondant à la désignation de la zone).

II. Formulation théorique du problème. - Soit donc une bande conductrice mince divisée en cinq zones électromagnétiques, rapportée à un système immobile de coordonnées cartésiennes Oxyz (Fig. 3) de base $\left(\mathbf{u}_{x}, \mathbf{u}_{y}, \mathbf{u}_{z}\right)$. Cette bande de longueur infinie (selon $O x$ ), de hauteur $2 a$ (selon $O y$ ) et de largeur $c$ (selon $O z$ ) est en mouvement de translation uniforme à la vitesse $\mathbf{V}=\mathbf{u}_{x} . V$ et coupe, sur une partie de sa longueur, matérialisée par les plans $x= \pm p \lambda / 2$ (Fig. 3), les lignes du champ magnétique d'excitation :

$$
\mathbf{B}_{\mathbf{c}}=\mathbf{u}_{y} \bar{B}_{\mathrm{c}} \mathrm{e}^{j(\omega t-k x)}
$$

avec :

$p$, nombre de paires de pôles, $\lambda$, longueur d'onde, $\bar{B}_{\text {c }}$, amplitude complexe et $k=2 \pi \lambda^{-1}$, nombre d'onde.

La bande est complétée sur toute sa longueur, de part et d'autre des plans $z= \pm c / 2$, par deux conducteurs immobiles - barres de fermeture - de même hauteur que la bande et de largeur $d$ (dans la direction $O z$ ) ; on néglige la résistance de passage entre les différentes zones de la bande et les barres de fermeture.

On considère la bande et les milieux environnants comme électriquement neutres au point de vue macroscopique ; de plus, la résistivité de la bande est suffisamment faible pour que les courants de déplacement puissent être négligés. Dans ces conditions, quatre expressions issues des équations de Maxwell et des équations caractéristiques du milieu suffisent pour définir le problème [1]:

$$
\begin{aligned}
\operatorname{rot} \mathbf{B} & =\mu \mathbf{J}_{\text {tot }} \\
\operatorname{rot} \mathbf{J} & =-\gamma\left[\frac{\partial \mathbf{B}}{\partial t}-\operatorname{rot} \mathbf{V} \wedge \mathbf{B}\right] \\
\operatorname{div} \mathbf{B} & =0 \\
\operatorname{div} \mathbf{J} & =0
\end{aligned}
$$

où $\mathbf{J}$ et $\mathbf{J}_{\text {tot }}$ représentent les vecteurs de la densité de courant, c'est-à-dire les grandeurs dont dérive le champ électromagnétique, et $\mathbf{B}$ le champ d'induction magnétique résultant.

Dans l'expression (1.1), la densité de courant total se répartit en deux composantes :

$$
\mathbf{J}_{\text {tot }}=\mathbf{J}_{\mathrm{c}}+\mathbf{J}
$$

$\mathbf{J}_{\mathbf{c}}$ est due aux sources extérieures à la bande (celles qui engendrent $\mathbf{B}_{\mathrm{c}}$ ) et $\mathbf{J}$ est induite à l'intérieur de la bande par le champ électromagnétique.

Nous ferons d'autre part les hypothèses suivantes :

- aucun échange d'énergie, par voie galvanique, n'est possible entre le système «bande-barres de fer- 
meture » et les milieux environnants supposés parfaitement isolants,

- la bande est suffisamment mince pour qu'aucune grandeur ne puisse varier sur sa hauteur $(\partial / \partial y \equiv 0)$; en particulier $\mathbf{J}_{y}=\mathbf{0}$,

- la perméabilité magnétique des milieux situés de part et d'autre de la bande, limités par les plans $y= \pm a$, est arbitrairement grande par rapport à celles de la bande elle-même et de l'espace environnant.

Le champ magnétique d'excitation est engendré ordinairement par des courants circulant dans les encoches d'une armature ferromagnétique (par exemple dans les bobines des inducteurs linéaires de la figure 1) ; pour le besoin des calculs, on remplace généralement la distribution discontinue des courants inducteurs par une distribution linéique équivalente. Dans le cas de notre modèle, cette dernière correspondrait à deux nappes filamentaires de courant, « acollées » à la bande dans les plans $y= \pm a$ et recouvrant toute la zone active III. Comme il est très malaisé d'opérer avec les densités linéiques de courant, nous répartirons par la suite la densité des courants d'excitation non pas dans les encoches des armatures ferromagnétiques (à cause de l'impossibilité d'une représentation mathématique générale), ni sur les nappes filamentaires, mais uniformément sur toute la hauteur de la bande. Il est équivalent, selon la démonstration ci-dessous et compte tenu des hypothèses faites, de considérer qu'il existe, à une abscisse $x$ quelconque une densité linéique de courant $\mathbf{I}_{c}$ dans une nappe d'épaisseur nulle ou une densité volumique de courant $\mathbf{J}_{c}=\mathbf{I}_{c} / 2$ a. En effet, le théorème d'Ampère :

$$
\oint_{\Gamma} \mathbf{H} \cdot \mathbf{d l}=\int_{S(\Gamma)} \mathbf{I}_{\mathbf{c}} \cdot \delta(y-a) \mathbf{d} \mathbf{s}
$$

où $S(\Gamma)$ est la surface limitée par la courbe fermée $\Gamma$ et $\delta$, l'opérateur de Dirac, s'écrit dans le plan $(x, y)$

$$
\begin{gathered}
\oint_{\Gamma} \mathbf{H} . \mathbf{d l}=\int_{-a}^{+a}\left[H_{y}(x+\Delta x, o, z)-H_{y}(x, o, z)\right] \mathrm{d} y \\
\int_{S(\Gamma)} \mathbf{I}_{\mathrm{c}} \cdot \delta(y-a) \mathbf{d} \mathbf{s}^{\prime}=I_{\mathrm{c}_{z}} \Delta x \int_{-a}^{+a} \delta(y-a) \mathrm{d} y \\
\lim _{\Delta x \rightarrow 0} \int_{-a}^{+a} \frac{H_{y}(x+\Delta x, o, z)}{\Delta x}-H_{y}(x, o, z) \\
\frac{\partial H_{y}}{\partial x} \int_{-a}^{+a} \mathrm{~d} y=I_{\mathrm{c}_{z}} \int_{-a}^{+a} \delta(y-a) \mathrm{d} y \\
\frac{\partial H_{y}}{\partial x}=\frac{I_{\mathrm{c}_{z}}}{2 a} .
\end{gathered}
$$

Un calcul analogue dans le plan $(y, z)$ conduit à :

$$
-\frac{\partial H_{y}}{\partial z}=\frac{I_{\mathbf{c}_{x}}}{2 a} \text {. }
$$

Les expressions (3) et $\left(3^{\prime}\right)$ sont les composantes de l'équation vectorielle

$$
\operatorname{rot} \mathbf{H}_{y}=\frac{\mathbf{I}_{\mathrm{c}}}{2 a}=\mathbf{J}_{\mathrm{c}} .
$$

Dans ces conditions, les relations (1.1) et (1.2) conduisent à l'équation équivalente

$$
\text { rot.rot }\left[\frac{\mathbf{J}}{\gamma}-\mathbf{V} \wedge \mathbf{B}\right]=-\mu \frac{\partial}{\partial t}\left(\mathbf{J}+\mathbf{J}_{\mathbf{c}}\right)
$$

dont le premier membre se décompose en deux termes :

$$
\begin{aligned}
\operatorname{rot} \cdot \operatorname{rot}\left(\frac{\mathbf{J}}{\gamma}-\mathbf{V} \wedge \mathbf{B}\right)=\operatorname{grad} \cdot \operatorname{div} & {\left[\frac{\mathbf{J}}{\gamma}-\mathbf{V} \wedge \mathbf{B}\right]-} \\
& -\nabla^{2}\left[\frac{\mathbf{J}}{\gamma}-\mathbf{V} \wedge \mathbf{B}\right] .
\end{aligned}
$$

Compte tenu de (3), (4) et de :

$\operatorname{div}(\mathbf{V} \wedge \mathbf{B})=\mathbf{B} \operatorname{rot} \mathbf{V}-\mathbf{V} \operatorname{rot} \mathbf{B}=-\mu \mathbf{V}\left(\mathbf{J}+\mathbf{J}_{\mathrm{c}}\right)$,

l'éq. (3) devient :

$$
\begin{array}{r}
\mu \operatorname{grad}\left[\mathbf{V} \cdot\left(\mathbf{J}+\mathbf{J}_{c}\right)\right]-\frac{1}{\gamma} \nabla^{2} \mathbf{J}+\nabla^{2}[\mathbf{V} \wedge \mathbf{B}]= \\
=-\mu \frac{\partial}{\partial t}\left(\mathbf{J}+\mathbf{J}_{\mathbf{c}}\right)
\end{array}
$$

En associant à cette équation, la relation $\operatorname{div} \mathbf{B}=0$, le système (1) devient dans le cadre de nos hypothèses :

$$
\left.\begin{array}{l}
\Delta J_{x}-\mu \gamma V \frac{\partial J_{x}}{\partial x}-\mu \gamma \frac{\partial J_{x}}{\partial t}=0 \\
\Delta J_{z}-\mu \gamma V \frac{\partial J_{z}}{\partial x}-\mu \gamma \frac{\partial J_{z}}{\partial t}=\mu \gamma\left[\frac{\partial J_{\mathrm{c}}}{\partial t}+V \frac{\partial J_{\mathrm{c}}}{\partial x}\right]
\end{array}\right\}
$$

où $J_{x}$ et $J_{z}$, composantes de $\mathbf{J}$ selon les axes $\mathbf{O x}$ et $\mathbf{O z}$, sont liés par (1.4).

L'intégration du système (6) permet de trouver la densité de courant induit $\mathbf{J}$ dans chacune des zones de la bande en fonction de la densité de courant extérieur d'excitation $\mathbf{J}_{\mathbf{c}}$. La résolution des équations complètes n'est évidemment nécessaire que dans la zone III ; pour les autres zones, on introduit les simplifications suivantes :

$$
\begin{aligned}
& \text { zones } \mathrm{I}, \mathrm{II}, \mathrm{IV}, \mathrm{V} ; \mathbf{J}_{c}=0, \\
& \text { zones } \mathrm{I}_{b}, \mathrm{II}_{b} \mathrm{IV}_{b}, \mathrm{~V}_{b} ; \mathbf{J}_{c}=0, \mathbf{V}=0 \\
& \text { zone } \mathrm{III}_{b} ; \mathrm{V}=0
\end{aligned}
$$

L'intégration du système (6) permet alors d'obtenir, à partir de (1.1), l'expression de $\mathbf{B}$ :

$B=\mu \int\left(-J_{x}\right) \mathrm{d} z+f(x)=\mu \int\left(J_{z}+J_{\mathrm{c}}\right) \mathrm{d} x+g(z)$.

Les conditions aux limites qu'il convient de respecter lors de la résolution de (6) et (7) et qui traduisent la continuité des composantes tangentielles du champ électrique et d'excitation magnétique, et la 
continuité de la composante normale de la densité volumique de courant, se résument ainsi :

- Entre les zones I et III au plan de jonction $x=(-p \lambda / 2) \forall z$ et entre les zones II et III au plan de jonction $x=+(p \lambda / 2) \forall z$

$$
\bar{E}_{z_{\mathrm{III}}}=\bar{E}_{z \mathrm{r}, \mathrm{II}} ; \quad \bar{H}_{y_{\mathrm{III}}}=\bar{H}_{y_{\mathrm{I}, \mathrm{II}}} ; \quad \bar{J}_{x_{\mathrm{III}}}=\bar{J}_{x_{\mathrm{I}, \mathrm{II}}}
$$

avec des conditions identiques pour les barres de fermeture.

- Entre les zones IV et I au plan de jonction: $x=-(p(\lambda / 2)+m) \forall z$ et entre les zones $\mathrm{V}$ et II au plan de jonction $x=(p(\lambda / 2)+m) \forall z$

$$
\bar{J}_{x_{\mathrm{I}, \mathrm{II}}}=\bar{J}_{x_{\mathrm{IV}}, \mathrm{v}} ; \quad \bar{E}_{z_{\mathrm{I}, \mathrm{II}}}=\bar{E}_{z_{\mathrm{IV}, \mathrm{V}}}
$$

avec des conditions identiques pour les barres de fermeture.

- Entre les barres et le conducteur massif, au plan de jonction $z=c / 2 \forall x$

$$
\bar{J}_{z_{L}}=\bar{J}_{z_{L_{b}}} ; \quad \bar{E}_{x_{L}}=\bar{E}_{x_{L_{b}}} ; \quad \bar{H}_{y_{L}}=\bar{H}_{y_{L_{b}}}
$$

avec $L=$ I, II, III, IV, V.

A ces conditions, il convient d'ajouter :

$$
J_{z_{L_{b}}}=0 \text { pour } z= \pm(c / 2+d) \quad \forall x .
$$

Remarquons que l'armature ferromagnétique ayant une perméabilité arbitrairement grande, l'excitation magnétique produite par les courants extérieurs reste confinée dans les zones couvertes par l'armature : c'est pourquoi l'excitation magnétique résultante n'est pas continue aux plans de jonction $x= \pm(p(\lambda / 2)+m)$, ce qui ne veut pas dire qu'elle est nulle. Tout se passe, pour le phénomène étudié comme si la perméabilité magnétique des zones IV et $\mathrm{V}$ et des barres correspondantes était nulle; ainsi on peut poser $\mu=0$ dans les zones $\mathrm{IV}, \mathrm{V}, \mathrm{IV}_{b}$ et $\mathrm{V}_{b}$.

III. Résolution du problème. - 1 . INTÉGRATION DU SYSTÈME (6) DANS LA BANDE CONDUCTRICE. - La symétrie du système par rapport au plan $x O y$ conduit à chercher une solution de l'équation sans second membre de la forme :

$$
\bar{J}_{z_{L}}^{\prime}=\sum_{i}\left(\bar{A}_{1 i_{L}} \exp \left(\bar{k}_{1 i_{L}} x\right)+\bar{A}_{2 i_{L}} \exp \left(\bar{k}_{2 i_{L}} x\right)\right) \operatorname{ch} \bar{\alpha}_{i_{L}} z
$$

où $\bar{k}_{1 i_{L}}$ et $\bar{k}_{2 i_{L}}$ sont solutions, pour $\bar{\alpha}_{i_{L}}$ donné, du polynôme de dérivation associé à (6), c'est-à-dire que

$$
\begin{gathered}
\bar{k}_{1,2 i_{L}}=\frac{k(1-g) R_{e m_{L}}}{2} \times \\
\times\left[1 \pm\left(1+j \frac{4}{(1-g)^{2} R_{e m_{L}}}-\left(\frac{2 k \bar{\alpha}_{i_{L}}}{(1-g) R_{e m_{L}}}\right)^{2}\right)^{1 / 2}\right]
\end{gathered}
$$

où le signe + est affecté à $\bar{k}_{1 i}$ et où

$$
g=1-(k V / \omega) \text { et } R_{e m}=\mu \omega \gamma \cdot k^{-2}
$$

représentant respectivement le glissement, c'est-à-dire la vitesse relative de la bande par rapport au champ extérieur, et le nombre de Reynolds magnétique.

Ainsi, compte tenu de (1.4), (8) et (9) la distribution finale des courants induits dans la $L$-ième zone est de la forme :

$$
\begin{gathered}
\bar{J}_{x_{L}}=\sum_{i}\left(-\alpha_{i_{L}}\right)\left(\frac{\left.\bar{A}_{1 i_{L}} \exp \left(\bar{k}_{1 i_{L}} x\right)+\frac{\bar{A}_{2 i_{L}}}{\bar{k}_{1 i_{L}}} \exp \left(\bar{k}_{2 i_{L}} x\right)\right) \operatorname{sh} \bar{\alpha}_{i_{L}} z}{\bar{J}_{i_{L}}}=\sum_{i}\left(\bar{A}_{1 i_{L}} \exp \left(\bar{k}_{1 i_{L}} x\right)+\bar{A}_{2 i_{L}} \exp \left(\bar{k}_{2 i_{L}} x\right)\right) \operatorname{ch} \bar{\alpha}_{i_{L}} z-\frac{j g R_{e m_{I I I}}}{1+j g R_{e m_{I I I}}} \cdot J_{c_{L}} \mathrm{e}^{-j k x} .\right.
\end{gathered}
$$

Le champ magnétique résultant le long de la bande, déduit de (10), (11) et (7), est

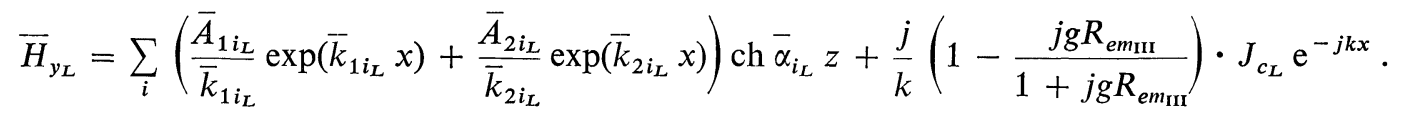

2. INTÉGRATION DU SYSTÈME (6) DANS LES BARRES DE FERMETURE. - La condition $J_{z_{L_{b}}}=0$ pour $z=(c / 2+d) \forall x$ conduit à chercher une solution de l'équation sans second membre sous la forme asymétrique, en procédant par la suite d'une façon identique à celle du paragraphe III.1, on obtient la distribution des courants et du champ magnétique circulant dans les barres de fermeture sous une forme analogue à (10), (11); les exponentielles correspondant à (9) se mettent toutefois sous la forme beaucoup plus simple :

$$
\bar{k}_{1,2 i_{L_{b}}}= \pm\left[j k^{2} R_{e m_{L_{b}}}-\bar{l}_{i_{L_{b}}}^{2}\right]^{1 / 2} .
$$

3. Application DeS CONDItions AUX Limites. Le modèle de la figure 3 met à notre disposition deux groupes de conditions aux limites :

- conditions entre la bande conductrice et les barres de fermeture,

- conditions entre les différentes zones longitudinales.

Le premier groupe permet d'établir la forme finale des expressions (10), (11). Ainsi les distributions des courants induits dans la bande et du champ magnétique résultant, s'écrivent après calculs : 


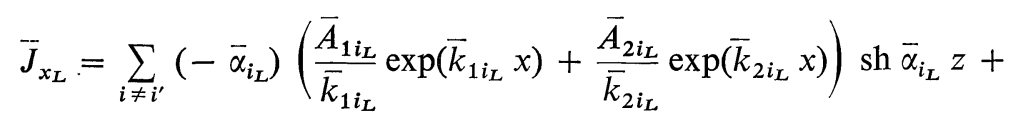

$$
\begin{aligned}
& +\frac{j \bar{\alpha}_{L}^{\prime}}{k}\left[\bar{J}_{z_{L}}^{\prime \prime}-\bar{J}_{z_{L_{b}}}^{\prime \prime}\left(1-\frac{1}{\operatorname{ch} \bar{l}_{L}^{\prime} d}\right)\right] \bar{K}_{L} \cdot \mathrm{e}^{-j k x} \cdot \operatorname{sh} \bar{\alpha}_{L}^{\prime} z . \\
& \bar{J}_{z_{L}}=\sum_{i \neq i^{\prime}}\left(\bar{A}_{1 i_{L}} \exp \left(\bar{k}_{1 i_{L}} x\right)+\bar{A}_{2 i_{L}} \exp \left(\bar{k}_{2 i_{L}} x\right)\right) \operatorname{ch} \bar{\alpha}_{i_{L}} z+ \\
& +\left[\bar{J}_{z_{L}}^{\prime \prime}\left(1-\bar{K}_{L} \operatorname{ch} \bar{\alpha}_{L}^{\prime} z\right)+\bar{J}_{z_{L_{b}}}^{\prime \prime}\left(1-\frac{1}{\operatorname{ch} \bar{l}_{L}^{\prime} d}\right) \bar{K}_{L} \operatorname{ch} \bar{\alpha}_{L}^{\prime} z\right] \mathrm{e}^{-j k x} . \\
& \bar{H}_{y_{L}}=\sum_{i \neq i^{\prime}}\left(\frac{\bar{A}_{1 i_{L}}}{\bar{k}_{1 i_{L}}} \exp \left(\bar{k}_{1 i_{L}} x\right)+\frac{\bar{A}_{2 i_{L}}}{\bar{k}_{2 i_{L}}} \exp \left(\bar{k}_{2 i_{L}} x\right)\right) \operatorname{ch} \bar{\alpha}_{i_{L}} z+ \\
& +\frac{j}{k}\left[\bar{J}_{z_{L}}^{\prime \prime}\left(1-\bar{K}_{L} \operatorname{ch} \bar{\alpha}_{L}^{\prime} z\right)+\bar{J}_{z_{L_{b}}}^{\prime \prime}\left(1-\frac{1}{\operatorname{ch} \bar{l}_{L_{b}}^{\prime} d}\right) \bar{K}_{L} \operatorname{ch} \bar{\alpha}_{L}^{\prime} z+J_{c_{L}}\right] \mathrm{e}^{-j k x}
\end{aligned}
$$

où les couples $\left(-j k, \bar{\alpha}_{L}^{\prime}\right)$ et $\left(-j k, \bar{l}_{L_{b}}^{\prime}\right)$ sont liés respectivement par les éq. (9) et (12) et avec :

$$
\bar{J}_{z_{L}}^{\prime \prime}=-j \frac{R_{e m_{L}}}{1+j g R_{e m_{L}}} J_{c_{L}}
$$

avec une expression analogue de $\bar{J}_{z_{L_{b}}}^{\prime \prime}$ dans laquelle évidemment $g=1$

$$
\bar{K}_{L}=\left(\operatorname{ch} \bar{\alpha}_{L}^{\prime} c / 2+\frac{\gamma_{L_{b}}}{\gamma_{L}} \cdot \frac{\bar{\alpha}_{L}^{\prime}}{\bar{l}_{L_{b}}^{\prime}} \operatorname{sh} \bar{\alpha}_{L}^{\prime} c / 2 \cdot \operatorname{th} \bar{l}_{L_{b}}^{\prime} d\right)^{-1} .
$$

Des expressions similaires à (13), (14) et (15), quoique plus complexes ont été établies pour les barres de fermeture.

Le deuxième groupe de conditions permet de déterminer les relations existant entre les amplitudes des grandeurs électromagnétiques des différentes zones. Compte tenu des formulations très proches des expressions des champs et des courants dans les cinq zones, et des exposants différents qu'elles comportent (ceux-ci imposés par les caractéristiques électrique, magnétique et géométrique propres à chacune d'elles) l'application des conditions aux limites aux plans de jonction nécessite une décomposition en séries de Fourier des diverses expressions obtenues, en utilisant l'extension périodique de celles-ci sur la largeur double, de l'ensemble " conducteur en mouvement - barres conductrices immobiles».

Par conséquent :

- Les courants $\bar{J}_{z_{L}}, \bar{J}_{z_{L_{b}}}$ et le champ résultant $\bar{H}_{y_{L}}$, $\bar{H}_{y_{L_{b}}}$ étant symétriques par rapport à $O x$, nous les décomposons en séries paires de la forme :

$$
\overline{f(x, z)}=\bar{A}_{0}(x)+\sum_{n} \bar{A}_{n}(x) \operatorname{ch} \bar{\beta}_{n} z
$$

avec $\bar{\beta}_{n}=-j(2 n+1) \pi /(c+2 d), n$, étant un nombre entier positif.

- Les courants $\bar{J}_{x_{L}}$ et $\bar{J}_{x_{L_{b}}}$ étant antisymétriques par rapport à $O x$, nous les décomposons en séries impaires de la forme :

$$
\overline{\varphi(x, z)}=\sum_{n} \bar{C}_{n}(x) \operatorname{sh} \bar{\beta}_{n} z .
$$

L'ensemble des conditions aux limites du deuxième groupe conduit à un système de 32 équations, qu'il serait trop fastidieux de reproduire dans le cadre de ce mémoire, et pour la solution duquel, nous disposons d'un programme en ALGOL.

IV. Analyse physique des phénomènes mis en évidence. - Le champ magnétique d'excitation (1.0), qui est à l'origine des phénomènes physiques liés à la bande en mouvement, et dont l'amplitude est constante sur toute la longueur de la zone active III, engendre les courants (13), (14) et le champ résultant (15), variables en $x$ et $z$. En effet, si l'on examine l'expression (15), on peut y distinguer deux systèmes d'onde : le premier, représenté par la combinaison polynomiale d'exponentielles complexes est, dans un cas général, un ensemble d'ondes amorties sur la longueur et la-largeur de la bande, qui se propagent à des vitesses différentes; le second, affecté du facteur $\mathrm{e}^{-j k x}$ symbolise les ondes glissant à la même vitesse que celles du champ d'excitation (1.0), mais dont une partie est amortie sur la largeur de la bande. Nous analysons ci-après le champ magnétique résultant de la zone III pour démontrer que sa déformation est due essentiellement à la géométrie de la bande, et en particulier à ses dimensions finies.

Etudions tout d'abord les deux termes sous le signe de la somme de (15) (elles représentent les ondes dont l'amortissement sur la longueur dépend du facteur $\bar{k}_{i}$ ). La relation (9) montre qu'au moins une des valeurs de $\bar{k}_{i_{\text {III }}}$ a une partie réelle non nulle. Si la zone III était 
de longueur infinie, il est facile de voir que l'exponentielle correspondante divergerait vers l'infini de même signe que la partie réelle de son exposant : ceci serait physiquement impossible et son amplitude serait nécessairement nulle. Si l'exposant était imaginaire pur, nous n'aurions aucune condition nous permettant de calculer son amplitude en fonction de celle de l'onde glissante initiale (1.0) (toujours dans le cas d'une zone III de longueur infinie) : cette onde ne pourrait donc être entretenue et son amplitude serait aussi nécessairement nulle. Il en résulte donc que toutes les ondes qui se superposent à l'onde glissante initiale sont dues à la longueur finie de la zone III.

Les termes entre crochets [ ] de (15) représentent en réalité deux ondes : l'une purement glissante $\left(\mathrm{e}^{-j k x}\right)$ se propageant selon $\mathbf{O x}$ et l'autre glissante et amortie sur la largeur $\left(\mathrm{e}^{-j k x} \cdot \mathrm{ch} \bar{\alpha}_{L}^{\prime} z\right)$ se propageant dans une direction différente. On remarque que l'amplitude de cette dernière dépend, par l'intermédiaire du facteur $\bar{K}_{L}(16)$, du rapport des conductibilités des barres et de la bande en mouvement ; le facteur $\bar{K}_{L}$ devient nul, soit lorsque $c$ tend vers l'infini (bande infiniment large), soit quand $\gamma_{\mathrm{II}_{b}}$ tend vers l'infini (cas théorique correspondant à une distribution de largeur infinie des courants induits dans la bande, à savoir $J_{x}=0$ ). Ainsi la déformation de l'onde progressive initiale est bien due à la largeur finie de la bande.

On peut saisir globalement le rôle de cette largeur finie lorsqu'on analyse les conditions aux limites entre la bande et les barres de fermeture, et en particulier la continuité de la composante tangentielle du champ électrique. Celle-ci conduit aux égalités :

$$
\begin{aligned}
& -\left(\gamma_{L}\right)^{-1} \bar{\alpha}_{i_{L}} \operatorname{sh} \bar{\alpha}_{i_{L}} c / 2=\left(\gamma_{L_{b}}\right)^{-1} \bar{l}_{1 i_{L_{b}}} \cdot \frac{\operatorname{ch} \bar{\alpha}_{i_{L}} c / 2}{\operatorname{th} \bar{l}_{1 i_{L_{b}}} d} \\
& -\left(\gamma_{L}\right)^{-1} \bar{\alpha}_{i_{L}} \operatorname{sh} \bar{\alpha}_{i_{L}} c / 2=\left(\gamma_{L_{b}}\right)^{-1} \bar{l}_{2 i_{L_{b}}} \cdot \frac{\operatorname{ch} \bar{\alpha}_{i_{L}} c / 2}{\operatorname{th} \bar{l}_{2 i_{L_{b}}} d} .
\end{aligned}
$$

Ces deux relations permettent de trouver, compte tenu des dimensions géométriques et des conductibilités des zones $L$ et $L_{b}$, quelles sont les ondes propres du système électromagnétique liées à ces deux zones : il y aura autant d'ondes que de solutions de (17) (il n'y a en effet aucune raison pour qu'un même $\bar{\alpha}_{i_{L}}$ vérifie les deux relations en même temps et inversement), mais pour un $\bar{\alpha}_{i_{L}}$ donné, l'une des deux amplitudes $\bar{A}_{1 i_{L}}$ ou $\bar{A}_{2 i_{L}}$ peut être nulle. On constate immédiatement que $\bar{\alpha}_{i L}$ est nul si l'on fait tendre $\gamma_{L_{b}}$ vers l'infini (c'est-à-dire en se plaçant dans un cas théorique équivalent à celui d'un conducteur massif de largeur infinie).

Nous voyons donc l'influence considérable des dimensions finies de la zone active de la bande massive. Celles-ci perturbent la propagation de l'onde principale entretenue et donnent, en plus, naissance à des ondes parasites, toutes ces ondes se réfléchissant sur les plans qui limitent la zone active ; ceci se complique du fait qu'il apparaît d'autres ondes « de fuite » dans les zones extérieures adjacentes à la zone active, elles- mêmes soumises à des réflexions sur les plans qui limitent leur zone de propagation.

En conclusion, il est impossible d'obtenir dans un conducteur de dimensions finies, une onde de champ magnétique résultant parfaitement glissante, sans utiliser de moyens particuliers pour y parvenir (nous n'avons considéré que le champ magnétique puisque c'est lui qui est commun aux deux milieux constitués par l'armature magnétique portant les courants d'excitation, et par le conducteur massif en mouvement, et que d'autre part, s'il ne comporte pas d'ondes parasites, les courants induits dans le conducteur n'en comportent pas non plus).

V. Obtention d'un champ magnétique résultant purement glissant dans la zone active III. - Le champ magnétique résultant (15) analysé dans le paragraphe précédent possède une forme compliquée très différente de celle du champ d'excitation (1.0). On peut alors se poser la question de savoir par quel moyen il est possible d'obtenir, dans la géométrie linéaire de la figure 1 ou 2, un champ magnétique résultant purement glissant. Si ceci était possible, il y aurait nécessairement une discontinuité aux extrémités de la zone III (en $x= \pm p \lambda / 2$ ), puisque de façon naturelle, le champ continu comporte des ondes parasites. Or, une telle discontinuité de la composante tangentielle du champ magnétique, à la jonction de deux milieux, impose l'existence d'une densité linéique de courants localisée à cette jonction. Dans notre cas, cette densité linéique, répartie sur toute la hauteur du conducteur apparaît, d'après le même raisonnement que celui qui a été fait au paragraphe II, comme équivalente à un courant localisé sur l'armature ferromagnétique aux extrémités de la nappe filamentaire.

Etant donné la symétrie en $z$ des amplitudes des ondes parasites, on peut essayer de trouver des courants localisés aux extrémités de la nappe tels que

$$
\begin{aligned}
& \text { en } \quad x=-p \lambda / 2: \bar{I}_{c_{\mathrm{I}}}=2 a\left[\bar{J}_{c_{\mathrm{I}}}+\bar{J}_{c_{\mathrm{I}}}^{\prime} \operatorname{ch} \theta z\right] \\
& \text { en } \quad x=+p \lambda / 2: \bar{I}_{c_{\mathrm{II}}}=2 a\left[\bar{J}_{c_{\mathrm{II}}}+\bar{J}_{c_{\mathrm{II}}}^{\prime} \operatorname{ch} \theta z\right] .
\end{aligned}
$$

Ces courants circulent sur toute la largeur de la carcasse $(c+2 d)$. Pour pouvoir calculer les amplitudes complexes de ces courants, il est nécessaire de décomposer les éq. (18) en série de Fourier comme on l'a déjà fait pour toutes les grandeurs électriques et magnétiques des différentes zones (cf. § III).

Nous aurons, dans la zone III :

$$
\bar{J}_{c_{\mathrm{I}}}=\frac{\bar{I}_{c \mathrm{I}}}{2 a}=\bar{b}_{0_{\mathrm{III}}}+\sum_{n} \bar{b}_{n_{\mathrm{III}}} \operatorname{ch} \bar{\beta}_{n} z
$$

et dans la zone $\mathrm{III}_{b}$ :

$$
\bar{J}_{c_{\mathrm{I}}}=\bar{b}_{\mathrm{OII}_{\mathrm{b}}}+\sum_{n} \bar{b}_{n_{\mathrm{II}_{b}}} \operatorname{ch} \bar{\beta}_{n} z
$$

L'introduction de ces densités linéiques aux extrémités des zones III et III $_{b}$ modifie alors les conditions aux limites relatives au champ magnétique résultant. 
En effet, la condition $\bar{H}_{y_{\mathrm{I}}}=\bar{H}_{y_{\mathrm{III}}}$ est alors remplacée par:

$$
\bar{H}_{y_{\mathrm{III}}}{ }^{-}-\bar{H}_{y_{\mathrm{I}}}=\bar{J}_{c_{\mathrm{I}}}+\bar{J}_{c_{\mathrm{I}}}^{\prime} \operatorname{ch} \theta z
$$

ce qui, décomposé en séries de Fourier, conduit à :

$$
\begin{aligned}
& \bar{B}_{0_{\mathrm{III}}}(-p \lambda / 2)+\sum_{n} \bar{B}_{n_{\mathrm{III}}}(-p \lambda / 2) \operatorname{ch} \bar{\beta}_{n} z- \\
& -\bar{B}_{0_{\mathrm{I}}}(-p \lambda / 2)-\sum_{n} \bar{B}_{n_{\mathrm{I}}}(-p \lambda / 2) \operatorname{ch} \bar{\beta}_{n} z= \\
& =\bar{b}_{0_{\mathrm{III}}}+\sum_{n} \bar{b}_{n_{\mathrm{III}}} \operatorname{ch} \bar{\beta}_{n} z .
\end{aligned}
$$

Par identification, on obtient finalement :

$$
\begin{aligned}
& \bar{B}_{0_{\mathrm{III}}}(-p \lambda / 2)-\bar{B}_{0_{\mathrm{I}}}(-p \lambda / 2)=\bar{b}_{0_{\mathrm{III}}} ; \\
& \bar{B}_{n_{\mathrm{III}}}(-p \lambda / 2)-\bar{B}_{n_{\mathrm{I}}}(-p \lambda / 2)=\overline{\bar{b}}_{n_{\mathrm{III}}}
\end{aligned}
$$

et de même entre les zones $I_{b}$ et $\operatorname{III}_{b}$ :

$$
\begin{aligned}
& \bar{B}_{0_{\mathrm{III}_{b}}}(-p \lambda / 2)-\bar{B}_{0_{\mathrm{III}_{b}}}(-p \lambda / 2)=\bar{b}_{0_{\mathrm{III}_{b}}} ; \\
& \bar{B}_{n_{\mathrm{II}_{b}}}(-p \lambda / 2)-\bar{B}_{n_{\mathrm{I}_{b}}}(-p \lambda / 2)=\bar{b}_{n_{\mathrm{III}_{b}}} .
\end{aligned}
$$

Le nouveau système des conditions aux limites comportant (19) et (20), conjugué avec l'hypothèse du champ magnétique purement glissant dans les zones III (et $\left.\mathrm{III}_{b}\right)$ (i. e. $\bar{A}_{i_{\text {III }}}=\bar{A}_{2 i_{\mathrm{III}}}=0 \forall i \neq i^{\prime}$ ) permet de calculer les amplitudes complexes des courants localisés aux plans de jonction $x= \pm p \lambda / 2$. Dans le cas général, le système obtenu est indéterminé ; c'est en effet un cas d'espèce : d'une part, il est nécessaire de connaitre le nombre de solutions de (17) ; d'autre part, ce système, qui est la traduction mathématique d'un certain nombre de conditions physiques, est nécessairement compatible, mais sa résolution n'est pas aisée ; l'opération ne pourra en fait être réalisée qu'expérimentalement, et plusieurs solutions sont possibles. Il y a cependant deux cas où le calcul est possible.

Le premier, d'intérêt théorique, est celui où les zones $L_{b}$ (barres de fermeture) ont une conductivité infinie $\left(\gamma_{L_{b}}=\infty\right)$ et où les zones de fuites I et II sont infiniment longues $(m=\infty)$. Dans ces conditions on déduit de (9), (16) et (17) $\bar{\alpha}_{i}=0$ et $\bar{K}_{L}=0$ et la solution des équations correspondantes conduit à :

$$
\begin{aligned}
& \bar{J}_{c_{\mathrm{I}}}=\frac{\left(\mu_{\mathrm{III}} / \mu_{\mathrm{I}}\right) \bar{k}_{1}+j_{k}}{k^{2}} \cdot \frac{J_{c} \mathrm{e}^{j k p \lambda / 2}}{1+j g R_{e_{m_{\mathrm{II}}}}} ; \\
& \bar{J}_{c_{\mathrm{II}}}=-\frac{\left(\mu_{\mathrm{III}} / \mu_{\mathrm{II}}\right) k_{2}+j k}{k^{2}} \cdot \frac{J_{c} \mathrm{e}^{-j k p \lambda / 2}}{1+j g R_{e_{m_{\mathrm{III}}}}} .
\end{aligned}
$$

Le deuxième cas, d'intérêt pratique, est celui où les zones $\mathrm{I}_{b}, \mathrm{II}_{b}, \mathrm{IV}_{b}$ et $\mathrm{V}_{b}$ sont inexistantes (ou ont une conductivité nulle). Dans ces conditions, la décomposition en séries de Fourier peut ne se faire que sur l'extension périodique de la largeur de la bande conductrice en mouvement (i. e. $\left.\bar{\beta}_{n}=-j(2 n+1) \pi / c\right)$. D'autre part, le système (17) montre que : $\bar{\alpha}_{i_{\mathrm{I}, \mathrm{II}}}=\bar{\beta}_{n}$.

Dans ces conditions, on obtient après quelques calculs identiques à ceux qui ont conduit à l'expression (21) :

$$
\begin{gathered}
\bar{J}_{c_{\mathrm{I}}}=\frac{j}{k} \mathrm{e}^{j k p \lambda / 2}\left\{J_{c}+\bar{J}_{\mathrm{ZII}_{\mathrm{II}}}^{\prime \prime}-\left[\bar{J}_{\mathrm{Z}_{\mathrm{III}}}^{\prime \prime}-\bar{J}_{\mathrm{Z}_{\mathrm{II}}}^{\prime \prime}\left(1-\frac{1}{\operatorname{ch} l^{\prime} d}\right)\right] \bar{K}_{\mathrm{III}} \cdot\left[\frac{\operatorname{sh} \bar{\alpha}^{\prime} c / 2}{\bar{\alpha}^{\prime} c / 2}-\operatorname{ch} \bar{\alpha}^{\prime} c / 2\left(\frac{8 \cdot S\left(\bar{\alpha}^{\prime}\right)}{c^{2}}-1\right)\right]\right\}- \\
-\frac{1}{\gamma_{\mathrm{III}} \mu_{\mathrm{III}} V}\left[\bar{J}_{\mathrm{Z}_{\mathrm{III}}}^{\prime \prime}-\bar{J}_{\mathrm{Z}_{\mathrm{II}}}^{\prime \prime}\left(1-\frac{1}{\operatorname{ch} \bar{l}^{\prime} d}\right)\right] \cdot \bar{K}_{\mathrm{III}} \mathrm{e}^{j k p \lambda / 2} \cdot \frac{\operatorname{sh} \theta c / 2}{\theta c / 2} \cdot \frac{\frac{4}{c} S\left(\alpha^{\prime}\right) \cdot \operatorname{ch} \bar{\alpha}^{\prime} c / 2-\frac{\operatorname{sh} \bar{\alpha}^{\prime} c / 2}{\bar{\alpha}^{\prime}}}{\frac{\operatorname{sh} \theta c / 2}{\theta}-\frac{4}{c} \operatorname{ch} \theta c / 2 \cdot S(\theta)} \\
\bar{J}_{c_{\mathrm{I}}}^{\prime}=\frac{1}{\gamma_{\mathrm{III}} \mu_{\mathrm{III}} V}\left[\bar{J}_{Z_{\mathrm{III}}^{\prime \prime}}-\bar{J}_{Z_{\mathrm{III}}}^{\prime \prime}\left(1-\frac{1}{\operatorname{ch} \bar{l}^{\prime} d}\right)\right] \bar{K}_{\mathrm{III}} \mathrm{e}^{j k p \lambda / 2} \cdot \frac{\frac{4}{c} \cdot S\left(\bar{\alpha}^{\prime}\right) \cdot \operatorname{ch} \bar{\alpha}^{\prime} c / 2-\frac{\operatorname{sh} \bar{\alpha}^{\prime} c / 2}{\bar{\alpha}^{\prime}}}{\frac{\operatorname{sh} \theta c / 2}{\theta}-\frac{4}{c} \operatorname{ch} \theta c / 2 \cdot S(\theta)}
\end{gathered}
$$

où

$$
S(\theta)=\sum_{n} \frac{1}{\theta^{2}-\bar{\beta}_{n}^{2}} \quad \text { et } \quad S\left(\bar{\alpha}^{\prime}\right)=\sum_{n} \frac{1}{\left(\bar{\alpha}^{\prime}\right)^{2}-\beta_{n}^{2}}
$$

sont des sommes finies.

Pour trouver les valeurs de $\bar{J}_{c_{\mathrm{II}}}$ et $\bar{J}_{c_{\mathrm{II}}}^{\prime}$ au plan de jonction $x=p \lambda / 2$, il suffit dans le système ci-dessus de changer : $\bar{J}_{c_{\mathrm{I}}}$ en $\left(-\bar{J}_{c_{\mathrm{II}}}\right) \bar{J}_{c_{\mathrm{I}}}^{\prime}$ en $\left(-\bar{J}_{c_{\mathrm{II}}}^{\prime}\right)$ et $(-p \lambda / 2)$ en $p \lambda / 2$ et l'on obtient finalement :

$$
\bar{J}_{c_{\mathrm{II}}}=-\bar{J}_{c_{\mathrm{I}}} \mathrm{e}^{-j k p \lambda} ; \bar{J}_{c_{\mathrm{II}}}^{\prime}=-\bar{J}_{c_{\mathrm{I}}}^{\prime} \mathrm{e}^{-j k p \lambda} .
$$

On remarque que les expressions (22) et (23) montrent que $\theta$ peut être quelconque, mais non nul, et que $\bar{J}_{c_{\mathrm{I}}}^{\prime}$ 
et $\bar{J}_{c_{\mathrm{II}}}^{\prime}$ ne sont nuls que si la conductivité de la zone III $_{b}$, $\gamma_{\mathrm{II}}$, est infinie (ou ce qui revient au même, la bande conductrice est infiniment large). On notera d'autre part que, $\bar{\alpha}^{\prime}$ n'étant jamais nul d'après (9), le champ magnétique est de la forme :

$$
\bar{H}_{y_{\mathrm{III}}}=\frac{j}{k}\left[\bar{J}_{\mathrm{Z}_{\mathrm{III}}^{\prime \prime}}\left(1-\bar{K}_{\mathrm{III}} \operatorname{ch} \bar{\alpha}^{\prime} z\right)+\bar{J}_{\mathrm{Z}_{\mathrm{II}}}^{\prime \prime}\left(1-\frac{1}{\operatorname{ch} \bar{l}^{\prime} d}\right) \cdot \bar{K}_{\mathrm{III}} \operatorname{ch} \bar{\alpha}^{\prime} z+J_{c}\right] \mathrm{e}^{-j k x}
$$

En conséquence, le champ magnétique (24) est la superposition d'une onde qui se propage selon $O X$, et d'une onde qui se propage dans une direction différente et qui est amortie sur la largeur de la zone III. Ce n'est que dans le cas où $\bar{K}_{\text {III }}=0$, c'est-à-dire le cas où $\gamma_{\mathrm{III}_{b}}$ est infinie que l'on a véritablement un champ magnétique purement glissant de forme comparable à (1.0).

VI. Conclusions. - En conclusion de cette étude", nous pouvons dire que, dans un conducteur massif de dimensions finies en mouvement de translation uniforme dans un champ magnétique initialement purement glissant, les champs magnétique et électrique qui existent à l'intérieur de ce conducteur ne sont pas des champs purement glissants, mais une superposition d'ondes glissantes plus ou moins amorties. Il est cependant possible de remédier à cet état de choses, dans la zone où le champ était initialement glissant, par une modification technologique du système source du champ magnétique initial: on notera toutefois que les grandeurs obtenues dans ces conditions ne comportent plus que l'onde initiale, affectée cependant d'un amortissement et d'une direction de propagation différente de la direction initiale, sauf dans un cas particulier, dont la réalisation bien que difficile à envisager est toutefois possible (barres de fermeture supraconductrices). Cette modification (étude $\mathrm{du} \S \mathrm{V}$ ) consiste en l'adjonction, aux extrémités de la zone active de deux enroulements créant un champ pulsatoire dont le déphasage par rapport au champ d'excitation principal est aisément calculable à l'aide de (22) et (23). Cette dernière partie peut être traitée dans un but un peu différent : on pourrait en effet penser à amortir plus ou moins les parasites existants au lieu de les annihiler complètement. Dans le cas d'un moteur linéaire, ceci doit nécessairement permettre de régler la vitesse de l'induit en mouvement en rapprochant plus ou moins le champ magnétique résultant du champ glissant idéal.

Remarquons enfin que l'étude de la répartition des champs et des courants dans un conducteur massif de dimensions finies en translation dans un champ glissant est en fait le problème théorique de fond de l'étude des machines tournantes et linéaires à induction (moteurs linéaires, pompes électromagnétiques, générateurs magnétohydrodynamiques à veine liquide), des relais de translation, des écrans magnétiques et autres systèmes électromécaniques soumis aux excitations alternatives. Elle peut déboucher sur la recherche des paramètres de construction de ces dispositifs. En effet, à partir des calculs qui ont été faits, on peut passer à ceux des puissances et partant, du rendement électrique.

Les auteurs tiennent à la disposition des lecteurs les détails des calculs intermédiaires qui n'ont pu être explicités dans le cadre de la présente publication.

\section{Bibliographie}

[1] AdLER (R. B.) et coll., " Electromagnetic energy transmission and Radiation ", John Wiley, 1966.

[2] KaNT (M.), « Equations générales du champ magnétique glissant d'une machine magnétohydrodynamique », C. R. Acad. Sci., Paris, 1968, Série A, 266, 164.
[3] Bonnefille (R.) et Kant (M.), «Application de la théorie du champ électromagnétique aux machines linéaires à induction », Revue, Phys. Appl., 1970, 5, 745 . 